\title{
La modalidad semipresencial y la pronunciación de la lengua inglesa: Resultados de un modelo apoyado con TIC
}

\section{Blended learning modality and English pronunciation: Findings from an ICT-supported model}

\author{
Ana M. Pinto-Llorente \\ Universidad Pontificia de Salamanca, Salamanca.España \\ ampintoll@upsa.es \\ M. Cruz Sánchez-Gómez \\ Universidad de Salamanca, Salamanca.España \\ mcsago@usal.es \\ Francisco J. García-Peñalvo \\ Universidad de Salamanca, Salamanca. España \\ fgarcia@usal.es \\ Marcos Cabezas-González \\ Universidad de Salamanca, Salamanca. España \\ mcabezasgo@usal.es
}

\begin{abstract}
Resumen
El objetivo de nuestro estudio fue examinar el potencial de los podcast, videocast, cuestionarios online, glosarios online y foros para aprender la pronunciación inglesa en un EVA implementado en modalidad semipresencial. La investigación se enmarca dentro de la metodología cuantitativa y se basa en la percepción de los estudiantes sobre estas tecnologías. La muestra estaba formada por 358 estudiantes matriculados en la asignatura de Inglés I. Los principales resultados del análisis descriptivo-inferencial llevado a cabo muestran su progreso en la pronunciación del inglés y en su capacidad para percibirlo y producirlo con mayor precisión. Su satisfacción hacia el curso fue positiva y su interés hacia la asignatura fue mayor con la aplicación de este innovador modelo. Consideran que el EVA les proporciona un ambiente más natural para practicar la pronunciación y adquirir las destrezas orales. Los datos muestran la eficacia y el potencial de estas herramientas tecnológicas para fines académicos, específicamente para el aprendizaje de la pronunciación inglesa y para desarrollar un aprendizaje autónomo. Nuestro estudio proporciona información para futuras propuestas educativas e innovadoras que nos permitirán decidir mejor las prioridades de intervención educativa y avanzar hacia modelos más eficaces para la enseñanza de la pronunciación inglesa en educación superior.
\end{abstract}

Palabras clave: pronunciación, tecnologías digitales, modalidad semipresencial, inglés como L2, aprendizaje autónomo

\begin{abstract}
Our goal in the present study was to examine the potential of podcast, videocast, online tests, online glossary and forums to learn English pronunciation in a VLE implemented on B-learning modality. The study is framed on the quantitative methodology and is based on students' perceptions of these technologies. The sample of our research was formed by 358 students enrolled in the subject of English I. The main results of the descriptive and inferential analysis performed show the students' progress in English pronunciation and the improvement of their ability to perceive and produce English more accurately. Their satisfaction towards the course was very positive and their interest towards the subject was higher with the implementation of this innovative
\end{abstract}


model. They consider that the VLE implemented supplied them with a more natural environment to practice pronunciation and acquire oral competences. Data show the effectiveness and potential for using these technological tools for academic purpose, specifically to learning English pronunciation and develop an autonomous learning. Our study provides more information for future educational and innovative proposals that will allow us to decide better the priorities in educational intervention and move towards more effective models to teach English pronunciation in higher education.

Keywords: pronunciation, technology, Blended learning, English as a L2, autonomous learning

\section{Introducción}

A lo largo de la historia de la enseñanza de la segunda lengua (L2) se han ido sucediendo diferentes métodos en los que la importancia que se le ha atribuido a la pronunciación ha ido variando de forma significativa tal y como Levis (2005) señala "The history of pronunciation in English language teaching is a study in extremes. (...) elevated pronunciation to a pinacle of importance (...) or ignored pronunciation."

En sus inicios la enseñanza de una lengua extranjera (LE) quedó básicamente relegada a la memorización de las normas gramaticales y listas de vocabulario. Con ello se pretendió capacitar al estudiante para leer textos, realizar traducciones y utilizar la L2 para comunicarse de forma escrita (Larsen-Freeman, 2003; Larsen-Freeman y Long, 1994; Sánchez, 1997). A esto se añadió el uso de la lengua materna de los estudiantes en todas las explicaciones con lo cual no existió una exposición oral a la L2 y, por supuesto, ningún interés por la pronunciación (Santos, 1999).

Opuesto al método gramática-traducción surgió el método directo que rechazó todas las ideas en las que se asentaba el método anterior y se convirtió en el primer enfoque en el que la enseñanza de un idioma era principalmente oral con la consiguiente mejora de las destrezas orales y el énfasis en una correcta pronunciación (Pastor, 2005). Surgió de un proyecto llevado a cabo por el alemán Maximilian Berlitz (1852-1921) en Rhode Island en 1978 y desde allí se extendió por toda Europa y América. Los principios y procedimientos seguidos en el método estaban muy determinados al uso exclusivo de la L2 en el aula, al aprendizaje del vocabulario y de las oraciones de la vida diaria en contexto, la gramática se enseñaba de forma inductiva y se ponían un gran énfasis en la pronunciación. Estos principios se reflejaban en las directrices seguidas en los colegios Berlitz (Richards y Rodgers, 2001)

A mediados del siglo XX surgió el método audio-oral con bases en la lingüística estructuralista, destacando a Bloomfield y a su escuela, y en la teoría conductista, con una influencia clara del psicólogo Skinner (1904-1990). Se trasladan las conclusiones que se habían extraído sobre la formación de hábitos, a través de la repetición de comportamientos en los animales, a las personas y a su vez a la adquisición de una L2 (Pastor, 2005; Sánchez, 1997). Fue el lingüista aplicado de origen español Robert Lado (1915-1995) el que en su obra titulada Language Teaching. A Scientific Approach (1964) presenta una clara descripción de este enfoque. Se consideraba que en la

La modalidad semipresencial y la pronunciación de la lengua inglesa: Resultados de un modelo apoyado con TIC. Ana $\mathrm{M}^{\mathrm{a}}$ Pinto, $\mathrm{M}^{\mathrm{a}}$ Cruz Sánchez, Francisco J. García-Peñalvo y Marcos Cabezas. 
enseñanza de L2 se debían potenciar las destrezas orales que se adquirían a través de la creación de hábitos y el aprendizaje de determinados patterns que se utilizaban de manera automática como respuesta a un estímulo. Se hacía un especial hincapié en la pronunciación y el error se corregía de forma inmediata para evitar que se adquiriera un hábito incorrecto.

Como señala Usó (2008) tras abandonar el método audio-oral, la pronunciación quedó relegada a un segundo plano y no fue hasta los años 90 cuando recuperó su importancia con el uso del método comunicativo, en el que la finalidad fundamental es la comunicación en la L2 y el cual tiene su base en la psicología cognitiva. Su objetivo es la adquisición de una adecuada competencia comunicativa que está conformada por un conjunto de competencias: gramatical, sociolinguiística, discursiva y estratégica, en las que se incluye una adecuada pronunciación. La acuñación del término competencia comunicativa se debe al antropólogo, lingüista y folclorista norteamericano Dell Hymes (1972), el cual considera que esta no hace referencia exclusivamente a la emisión de enunciados gramaticalmente correctos, sino que en la comunicación hay que tener en cuenta los aspectos del contexto social y cultural en el que tiene lugar. Por lo tanto, se hace imprescindible conocer al emisor, el momento en que tiene lugar la comunicación o el lugar en el que se desarrolla. Posteriormente Canale (1983) y Canale y Swain (1980) realizan una definición de competencia comunicativa, a la que nos suscribimos, como aquella en la que se interrelacionan cuatro competencias: competencia lingüística (también conocida como competencia gramatical), competencia sociolingüística, competencia discursiva y competencia estratégica. En 2001, durante el Año Europeo de las Lenguas y tras más de diez años de trabajo, el Consejo Europeo (2001) publica el Marco Común Europeo de Referencia para las Lenguas: aprendizaje, enseñanza, evaluación (MCER), el cual forma parte del proyecto general de su política lingüística que se ejemplificaba en el simposio de Rüschlikon (Suiza) titulado Transparencia y coherencia en la enseñanza en Europa: objetivos, evaluación, certificación. En este documento se considera que la competencia comunicativa está compuesta por: las competencias lingüísticas, las competencias sociolingüísticas y las competencias pragmáticas. En el método comunicativo la pronunciación desempeña un papel fundamental en relación a la destrezas orales, convirtiéndose en un aspecto esencial a la hora de determinar la competencia comunicativa de un hablante de L2 (Iruela, 2007). Es básica para la interacción, ya que si la pronunciación del hablante es adecuada, no existirán obstáculos en la comunicación y se producirá una mayor aceptación por parte de los hablantes nativos (Cortés, 2002). Como Bartolí (2005) indica en su trabajo sobre La pronunciación en la clase de lenguas extranjeras el hecho de que el estudiante tenga una adecuada pronunciación es un aspecto que se elogia y contribuye a que el discente tenga una mayor autoestima y confianza. Es por lo tanto necesario que la pronunciación reciba la importancia y el tratamiento adecuado, y que se consiga hacer un mayor hincapié en ella, ya que "la pronunciación no ha seguido el mismo camino que otras áreas de la enseñanza comunicativa de lenguas, como la gramática o la expresión escrita" (Iruela, 2004). No se ha conseguido dedicarle la misma atención que a otros aspectos de la L2 al desconocerse la manera de integrarla en el enfoque comunicativo, ya que "(...) teachers are often left to rely on their own intuitions with little direction"

La modalidad semipresencial y la pronunciación de la lengua inglesa: Resultados de un modelo apoyado con TIC. Ana $\mathrm{M}^{\mathrm{a}}$ Pinto, $\mathrm{M}^{\mathrm{a}}$ Cruz Sánchez, Francisco J. García-Peñalvo y Marcos Cabezas. 
(Derwing y Munro, 2005), y al no contar con materiales o herramientas que favorezcan esta integración (Bartolí, 2005). Esta última afirmación nos llevó a plantearnos la siguiente investigación, ya que consideramos que la tecnología podía ayudar a la integración de la pronunciación en el proceso de enseñanza-aprendizaje de la L2. En concreto nos planteamos las posibilidades que podría ofrecer un Entorno virtual de aprendizaje (EVA) al desafío que supone la aproximación a la pronunciación de la L2 y a su aprendizaje en la modalidad semipresencial. El modelo implementado se convierte en un intento de fomentar la enseñanza de la pronunciación del inglés como L2 para conseguir que cuente con la misma práctica que el resto de las competencias.

En las últimas décadas, gracias al rápido desarrollo que se ha producido en las diversas tecnologías, se ha potenciado la convergencia de los ámbitos de enseñanza-aprendizaje de la educación tradicional, en la que se llevan a cabo interacciones face-to-face, y de la educación mediada a través de la tecnología (Coaten, 2003; Graham, 2006; López y Matesanz, 2009).

Graham (2006:6. Figura 1) muestra que se ha producido una progresiva convergencia entre los dos sistemas de formación, presencial (face-to-face) y en línea, que han permitido que la conjunción de ambos, dé lugar al blended learning (García-Peñalvo y Seoane-Pardo, 2015; Gaercía-Peñalvo, 2015; Gros y García-Peñalvo, 2017)).

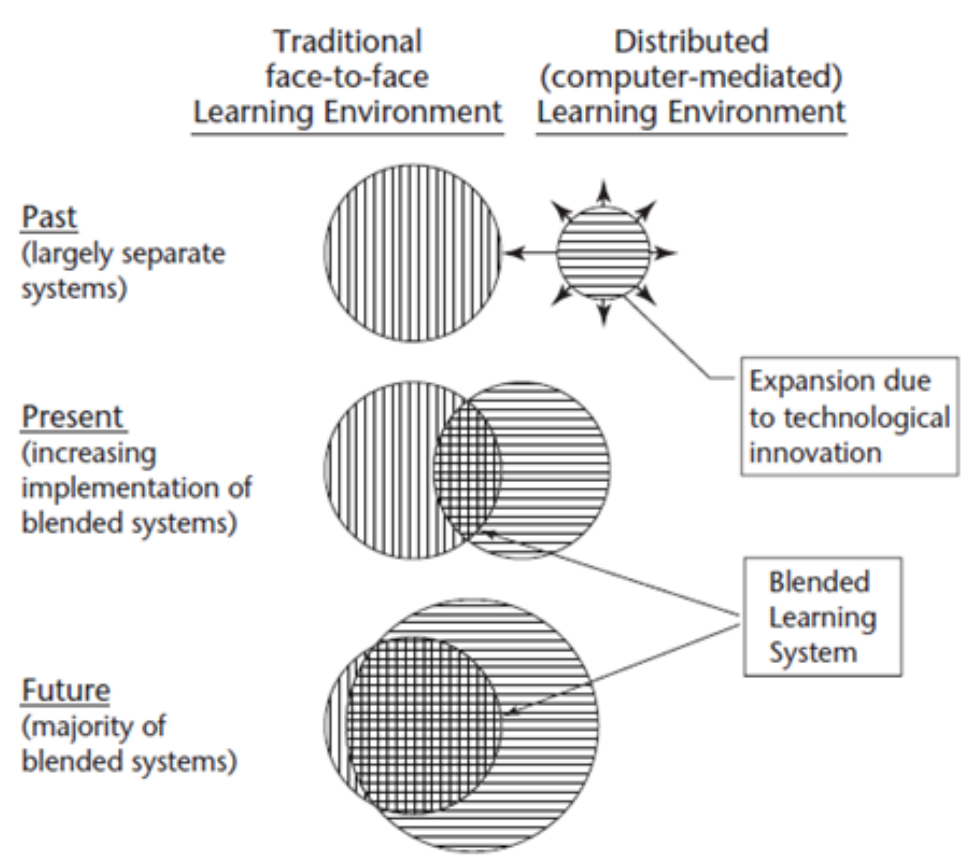

Figura 1. Convergencia entre los sistemas de formación presencial y en línea (Graham, 2006)

La consecuencia directa de esta convergencia entre ambos ámbitos de enseñanza es el desarrollo de ambientes en los que se aprovechan las ventajas de ambas modalidades de enseñanza (Graham, 2006; Osguthorpe y Graham, 2003). Como indica Thorne (2003):

La modalidad semipresencial y la pronunciación de la lengua inglesa: Resultados de un modelo apoyado con TIC. Ana $\mathrm{M}^{\mathrm{a}}$ Pinto, $\mathrm{M}^{\mathrm{a}}$ Cruz Sánchez, Francisco J. García-Peñalvo y Marcos Cabezas. 
"It represents an opportunity to integrate the innovative and technological advances offered by online learning with the interaction and participation offered in the best of traditional learning. It can be supported and enhanced by using the wisdom and one-to-one contact of personal coaches."

De esta forma, la modalidad blended learning, consigue combinar la interacción cara a cara que tiene lugar entre el profesor y los estudiantes en la modalidad presencial, que se consigue por la coincidencia en el tiempo y en el espacio de todos los protagonistas implicados, con la formación a través del uso de diferentes tecnologías que facilitan la distribución de contenidos, las experiencias de aprendizaje y la interacción sin que se precise que los discentes y los docentes compartan el mismo espacio físico y coincidan en el tiempo. Sin embargo, autores como Dzuiban, Hartman y Moskal (2004) no consideran que la modalidad blended learning sea, únicamente, el uso de distintas modalidades que se juntan y dan como resultado una modalidad nueva, sino que el blended learning debe convertirse en un enfoque pedagógico que destaque por el aprendizaje activo y colaborativo que nos brindan las tecnologías y la efectividad y socialización de la interacción face-to-face de la enseñanza presencial. Es decir:

"The real importance and significance in blended learning lies in its potential. If we forget the title and focus on the process, blended learning represents a real opportunity to create learning experiences that can provide the right learning at the right time and in the right place for each and every individual, not just at work, but in schools, universities and even at home. It can be truly universal, crossing global boundaries and bringing groups of learners together through different cultures and time zones. In this context blended learning could become one of the "most significant developments of the 21 st century." (Thorne, 2003)

Otros autores como Allan (2007); Sharpe, Benfield, Roberts y Francis (2006) en lugar de dar una definición del blended learning, prefieren señalar las dimensiones que abarca esta modalidad, es decir:

- $\quad$ uso de diferentes modos de enseñanza presencial, face-to-face, y en línea;

- mezcla de tecnologías basadas en la red. Uso de las tecnologías de la primera generación de Internet, pero también de las proporcionadas por el desarrollo tecnológico y por la Web 2.0;

- actividades e intervenciones bien de forma sincrónica como asincrónica. Flexibilidad de tiempo. Ruptura de las barreras temporales;

- el contexto donde se produce el aprendizaje es diverso, puede ser en el campus universitario, pero también en casa o en lugar de trabajo. Flexibilidad con respecto al lugar. Ruptura de las barreras espaciales;

- diferentes enfoques de aprendizaje. Pedagogías centradas en el tutor (tutorcentred) $\mathrm{o}$ en el estudiante (learner-centred). Teorías conductistas o constructivistas;

La modalidad semipresencial y la pronunciación de la lengua inglesa: Resultados de un modelo apoyado con TIC. Ana $\mathrm{M}^{\mathrm{a}}$ Pinto, $\mathrm{M}^{\mathrm{a}}$ Cruz Sánchez, Francisco J. García-Peñalvo y Marcos Cabezas. 
- la relación con otros en el proceso de aprendizaje es diverso. Aprendizaje individual, en grupo, pudiéndose desarrollar una comunidad de aprendizaje, aprendizaje colaborativo.

- modalidad dirigida a diferentes grupos de personas, bien estudiantes, profesionales, etc.; y

- los docentes determinan los objetivos del proceso de aprendizaje o pueden negociarse y acordarse por los protagonistas: los individuos, el grupo o las comunidades.

El blended learning se presenta como la manera idónea de combinar lo mejor que se nos ofrece desde otras modalidades y al mismo tiempo contar con la flexibilidad del tiempo, el espacio y los distintos enfoques de aprendizaje, así como de distintas tecnologías para tener la libertad de elegir aquellas que nos sean necesarias para el diseño de un determinado programa semipresencial, de acuerdo a los estudiantes o usuarios que tengamos (Allan, 2007). Además la flexibilidad en el tiempo y en el espacio permite que los discentes puedan tener libertad a la hora de decidir el momento en el que se conectan y el lugar desde el que lo hacen (Rosenberg, 2001). Esto hace que estemos ante una modalidad que posibilita que el estudiante marque su propio ritmo de aprendizaje de acuerdo a su situación personal.

\section{Modelo}

El modelo implementado para la asignatura de Idioma I (inglés) se diseñó a través de la plataforma de software libre Moodle. Esta destaca por contar con una comunidad de apoyo y desarrollo, y por basarse en una filosofía educativa sólida (Cole y Foster, 2008). Es decir, una plataforma para la creación y gestión de cursos basados en Internet. Que proporciona a los educadores las herramientas necesarias para crear un curso en un lugar web y además permitir el acceso a los estudiantes inscritos o matriculados en este. Permite el acceso a una gran cantidad de herramientas que hacen al curso más efectivo. Es un proyecto inspirado en la pedagogía construccionista social (Rice, 2011) y esto lo distingue de otros CMS (Content Management System), pues la mayoría de ellos se desarrollan alrededor de una serie de herramientas y no en torno a la pedagogía ni a ninguna teoría educativa.

El diseño parte de la selección de un modelo pedagógico de acuerdo a los principios de un ambiente de aprendizaje constructivista basado en la web (Duffy y Cunningham, 1996; Honebein, 1996; y Knuth y Cunnigham, 1993) en el que los estudiantes están situados en el centro del proceso de enseñanza-aprendizaje.

El formato de presentación de los materiales y actividades elegido en el diseño es el de temas. En el primero de ellos se presenta toda la información relativa al nombre de la asignatura, al docente que la imparte, al horario de tutorías virtuales y presenciales. En este mismo bloque también aparece la guía de aprendizaje.

El resto de los temas que conforman el modelo se estructuran en torno a los contenidos de la asignatura, partiendo de los más sencillos a los más complejos, para así establecer

La modalidad semipresencial y la pronunciación de la lengua inglesa: Resultados de un modelo apoyado con TIC. Ana $\mathrm{M}^{\mathrm{a}}$ Pinto, $\mathrm{M}^{\mathrm{a}}$ Cruz Sánchez, Francisco J. García-Peñalvo y Marcos Cabezas. 
una progresión en su dificultad. En cada uno de estos temas los estudiantes cuentan con recursos transmisivos como archivos en distintos formatos documentos de Word, PDF, podcast y videocast, así como enlaces a las páginas web en las que los estudiantes tienen acceso a diccionarios de referencia, páginas de prestigio para la enseñanza del inglés o páginas en las que los discentes tienen acceso a software para la creación de sus propios podcast. Además los podcast y videocast disponibles en el modelo podían descargarse para que los discentes pudieran escucharlos y trabajar con ellos en diferentes soportes como sus smartphones, tables u ordenadores. Este hecho facilitó el acceso a los contenidos de la asignatura y a la práctica de los mismos en multitud de situaciones, contextos y de acuerdo a las necesidades de los estudiantes (Man-Man, 2006). Los temas o bloques de contenido presentan también recursos interactivos como el glosario y los cuestionarios online. En el caso del glosario online se configuró esta herramienta para que fueran los estudiantes los únicos creadores de la actividad. Se les proporcionó un tutorial sobre su uso y se les indicaron los criterios de evaluación de la actividad. Por otro lado, los cuestionarios online se diseñaron para la realización de actividades de evaluación y autoevaluación. En el primer caso, se habilitaron los cuestionarios con un tiempo limitado, permitiendo un único intento y aplicando penalizaciones en las respuestas incorrectas. Por otro lado, los cuestionarios de autoevaluación estaban disponibles desde principios de curso, los estudiantes podían realizarlos en el momento que lo consideraran necesario y el número de veces que precisaran. La finalidad de estos era ayudar a los discentes a preparar los contenidos de la asignatura de cara a la realización de los cuestionarios evaluables y el examen final, y que los estudiantes conocieran su nivel y necesidades. En ambos tipos de cuestionarios (de evaluación y autoevaluación) se incluía la retroalimentación. Además los discentes contaban con un guía de uso de estas herramientas. En el modelo implementado los alumnos también contaron con recursos colaborativos como los foros que se dividieron en dos tipos. Por un lado, se habilitaron foros con la finalidad de fomentar la interacción entre los estudiantes para que pudieran preguntar sus dudas y ayudarse en su resolución, de esta forma se pretendía facilitar la comunicación y crear una comunidad de aprendizaje. Por otro lado, se presentaron foros cuya finalidad era facilitar la realización de actividades en grupo que posteriormente eran evaluadas por el profesor. Los estudiantes conocían desde principios de curso los foros que se iban a tener lugar para la evaluación de la asignatura juntos con los criterios que el docente iba a seguir. Además se les proporcionó una guía de uso de esta herramienta.

Este artículo tiene como objetivo presentar los resultados de un estudio descriptivo e inferencial sobre el potencial que ofrecen las herramientas señaladas anteriormente: podcast, videocast, cuestionarios online, glosario online y foros en la pronunciación del inglés como L2. El artículo se organiza en tres apartados. En el primero de ellos se realiza la exposición del método utilizado, en el que se incluye el modelo de investigación, los objetivos e hipótesis, población y muestra, instrumentos y variables, el trabajo de campo y la datación, organización y análisis de los datos recogidos. En el segundo apartado se exponen los resultados obtenidos en la investigación. Y se finaliza con la exposición de la discusión de los resultados.

La modalidad semipresencial y la pronunciación de la lengua inglesa: Resultados de un modelo apoyado con TIC. Ana $\mathrm{M}^{\mathrm{a}}$ Pinto, $\mathrm{M}^{\mathrm{a}}$ Cruz Sánchez, Francisco J. García-Peñalvo y Marcos Cabezas. 


\section{Método}

Nuestra investigación es de carácter cuantitativo y se enmarca dentro de lo que en investigación educativa se denomina diseño no experimental o ex-post-facto. En concreto se define como aquella que se lleva a cabo sin que los investigadores manipulen las variables independientes deliberadamente. No se produce ninguna intervención sobre estas, ya que tanto ellas como sus efectos ya se han sucedido. El investigador inicia el estudio observando las variables dependientes, los fenómenos tal y como suceden en un determinado contexto de forma natural, y registra sus medidas para posteriormente llevar a cabo el análisis de las mismas. Se caracteriza por relacionar variables que no han sido manipuladas, de manera que una relación entre ellas no puede ser interpretada en sentido causal (Hernández, Fernández y Baptista, 2010; Kerlinger, 1985). En nuestra investigación hemos estudiado un grupo natural ya formado, compuesto por estudiantes que cursan en modalidad semipresencial el Grado en Maestro en Educación Primaria en la Mención de Lengua Extranjera Inglés, en concreto la asignatura de Idioma I (inglés). En este grupo lógicamente no hemos podido manipular las variables que determinan su opinión sobre el modelo tecnológico implementado en modalidad semipresencial, su conocimiento de la lengua inglesa y su uso de las distintas herramientas tecnológicas.

Los trabajos empíricos no experimentales presentan diferentes diseños. Siguiendo la clasificación de Arnal, Rincón y Latorre (1994) distinguimos los diseños comparativocausales que permiten planificar estudios para descubrir relaciones causa-efecto entre variables; los diseños correlacionales que se utilizan para determinar la relación entre variables y detectar la variabilidad de una variable que ha sido explicada por otra; y los diseños descriptivos que permiten describir un fenómeno, analizar su estructura y buscar relaciones entre las características que lo definen. En concreto en este último diseño se incluirían el estudio de desarrollo, cuyo objetivo es conocer los cambios que se producen en los sujetos con el transcurso del tiempo; el de método de encuesta, orientado a describir una situación; el estudio de casos, centrado en describir y analizar entidades o unidades educativas únicas; y el método observacional, que se caracteriza porque la información se recoge directamente tras la observación de los sujetos. Dentro de los métodos descriptivos se distinguen cuatro tipos (Colás y Buendía, 1998): método encuesta, orientado a la descripción de una situación dada; estudio analítico en el que el fenómeno es analizado en términos de sus componentes básicos; estudio observacional caracterizado porque la información es recogida de forma directa de los sujetos observados y no mediante respuesta de ellos; y estudio sobre el desarrollo que tiene como principal objetivo investigar patrones y secuencias de desarrollo y/o cambio como una función del tiempo.

En nuestra investigación se aborda un estudio descriptivo por encuesta, empleando técnicas de análisis descriptivo e inferencial para los distintos estratos muestrales considerados en el estudio. Con esta metodología de investigación, queremos dar respuesta a nuestros objetivos tanto en términos descriptivos como de relación y

La modalidad semipresencial y la pronunciación de la lengua inglesa: Resultados de un modelo apoyado con TIC. Ana $\mathrm{M}^{\mathrm{a}}$ Pinto, $\mathrm{M}^{\mathrm{a}}$ Cruz Sánchez, Francisco J. García-Peñalvo y Marcos Cabezas. 
comparación entre variables tras una recogida de información sistemática, según un diseño previamente establecido que asegura el rigor de la información obtenida.

Como Van Dalen y Meyer (1981) señalan, estos métodos resultan muy apropiados en determinados campos educativos, facilitando: recoger información factual detallada que describa una determinada situación; identificar problemas; realizar comparaciones y evaluaciones; y planificar futuros cambios y tomas de decisiones.

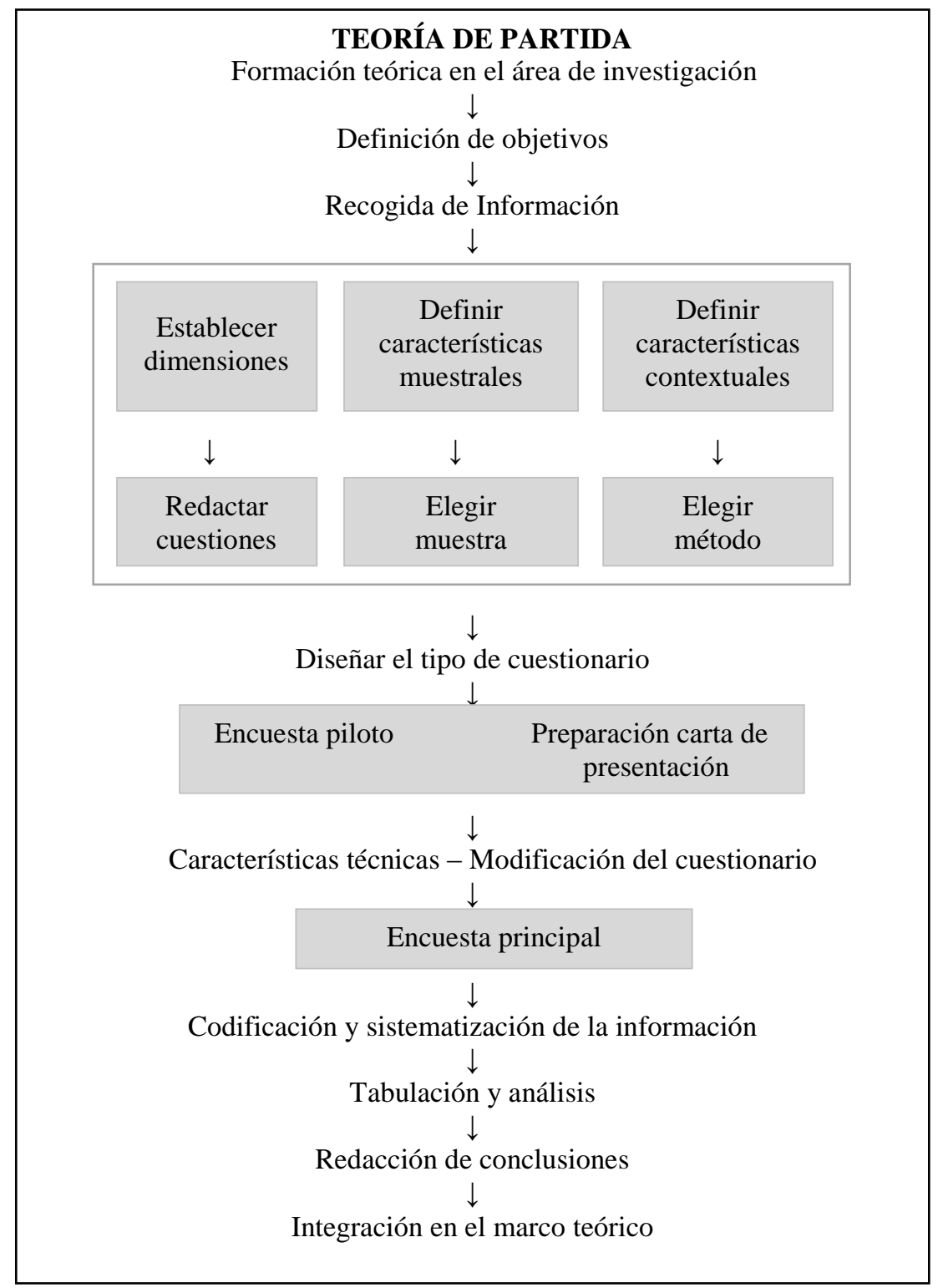

Figura 2. Fases de la Investigación por encuesta (Adaptado de Buendía, Colás y Hernández, 1997)

La modalidad semipresencial y la pronunciación de la lengua inglesa: Resultados de un modelo apoyado con TIC. Ana $\mathrm{M}^{\mathrm{a}}$ Pinto, $\mathrm{M}^{\mathrm{a}}$ Cruz Sánchez, Francisco J. García-Peñalvo y Marcos Cabezas. 
Para el diseño de nuestra investigación por encuesta hemos tomado como referencia las tres fases que establecen Buendía, Colás y Hernández (1997:121) (Figura 2) y que se resumen en:

1. Teórico-conceptual: planteamiento de objetivos y/o problemas e hipótesis de investigación

2. Metodológica: selección de la muestra y de las variables objeto de estudio; confección del cuestionario piloto y su formulación definitiva.

3. Estadística-conceptual: codificación y análisis de datos para obtener resultados a partir de los cuales se podrán realizar generalizaciones, e integrar en el marco teórico de partida las conclusiones elaboradas.

\section{Objetivos e hipótesis}

El objetivo de este estudio es poner de relieve el potencial pedagógico de herramientas transmisivas, interactivas y colaborativas: podcast, videocast, cuestionarios en línea, glosario en línea y foros en el aprendizaje de la pronunciación de la lengua inglesa.

Para investigar este ámbito, hemos sugerido las siguientes hipótesis:

H1. La mejora de la pronunciación de la lengua inglesa de nuestros estudiantes es mayor con la aplicación de las herramientas propuestas en el modelo tecnológico.

$\mathrm{H} 2$. El EVA implementado favorece el uso de herramientas transmisivas, interactivas y colaborativas para el aprendizaje de la pronunciación de la lengua inglesa y para desarrollar su competencia para percibir y producir el inglés con mayor precisión.

H3. La mejora del nivel de los estudiantes de sus destrezas orales y de la pronunciación de la lengua inglesa se debe a la eficacia de las actividades propuestas en el modelo implementado en la asignatura de Idioma I (inglés).

\section{Población y muestra}

Tomando como criterio el objetivo de nuestra investigación la población estaría conformada por los estudiantes del Grado en Maestro en Educación Primaria en la Mención de Lengua Extranjera Inglés de la modalidad semipresencial de la Facultad de Educación de la Universidad Pontificia de Salamanca. En concreto los estudiantes matriculados en la asignatura de Idioma I (inglés) que forma parte del Plan de estudios y que se imparte en el tercer curso. Dicha asignatura tiene carácter opcional y una carga lectiva de 6 ECTS (European Credit Transfer and Accumulation System) donde cada ECTS equivale a 25 horas de enseñanza. De acuerdo con los datos facilitados por la Secretaría de la Universidad la población estaría constituida por 451 estudiantes.

Por su parte la muestra de nuestra investigación sería una muestra probabilística, ya que todos los integrantes de la población tienen las mismas posibilidades de ser escogidos y además se quiere que los resultados puedan ser generalizados a una población. El tipo de muestreo realizado fue por conglomerados, ya que los individuos de la investigación formaban agrupaciones naturales. La muestra final estuvo conformada por 358 sujetos.

La modalidad semipresencial y la pronunciación de la lengua inglesa: Resultados de un modelo apoyado con TIC. Ana $\mathrm{M}^{\mathrm{a}}$ Pinto, $\mathrm{M}^{\mathrm{a}}$ Cruz Sánchez, Francisco J. García-Peñalvo y Marcos Cabezas. 
Para calcular el tamaño muestral necesario para que fuera una muestra probabilística representativa, utilizamos la fórmula propuesta por Cea D'ancona (1998:170).

$$
n=\frac{Z^{2} \hat{P} \hat{Q} N}{E^{2}(N-1)+Z^{2} \hat{P} \hat{Q}}=\frac{2^{2} \times 50 \times 50 \times 451}{\left(2,5^{2} \times 450\right)+(4 \times 50 \times 50)}=352
$$

Al contar con una muestra de 358 sujetos, se garantiza el tamaño mínimo exigido con un margen de error del 2,5\%, cumpliendo los requisitos de representatividad para continuar el trabajo de investigación.

Los participantes tenían una edad entre 20 y 58 años. Para el análisis de los datos obtenidos en la investigación se dividieron los estudiantes en cuatro grupos. El 24,6\% pertenecían al grupo de edad entre 20 y 24 años, el 25,7\% al grupo de 25 a 29 años, el $34,4 \%$ al de 30 a 34 y el 15,4\% al grupo de 35 o más de 35 años. En cuanto al sexo de los participantes, el 78,1\% eran mujeres mientras que el $21,9 \%$ hombres.

Todos los estudiantes que participaron en la investigación tenían una titulación universitaria previa que, en su mayoría, estaba relacionada con la educación $(93,3 \%)$ y gran parte de ellos $(84,7 \%)$ se habían incorporado al mercado laboral. El $27,7 \%$ trabajaba a tiempo parcial y el $57 \%$ a tiempo completo. Dentro del trabajo desempeñado por estos sujetos el 71,5\% tenía uno relacionado con la educación, principalmente eran maestros en la etapa de educación primaria $(62,6 \%)$.

La mayor parte de ellos $(99,2 \%)$ contaba con ordenador en su domicilio y más de la mitad $(68,4 \%)$ solían utilizarlo a diario y entre $10(56,7 \%)$ y $20(25,7 \%)$ horas a la semana. En cuanto al uso de internet, el 98,6\% cuenta con conexión en su domicilio y suele utilizarlo a diario $(63,1 \%)$, en su mayoría, entre $10(69 \%)$ y $20(21,2 \%)$ horas a la semana. Lo utilizan principalmente para buscar información (83\%), para comunicarse (91\%) y como ocio (71\%). Menos de la mitad de los sujetos (42\%) aseguraron utilizarlo como herramientas de formación.

\section{Instrumento y variables}

El instrumento utilizado en la presente investigación para operativizar las variables y recoger los datos fue el cuestionario. Como Colás y Buendía señalan (1998) "Con él se pretende conocer lo que hacen, opinan o piensan los encuestados mediante preguntas realizadas por escrito y que pueden ser respondidas sin la presencia del encuestador".

El diseño de los cuestionarios se realizó en tres fases. En la primera de ellas, se diseñaron los ítems que se iban a incluir en el cuestionario, tomando diferentes decisiones sobre la relación de las distintas preguntas con cada una de las dimensiones a conocer. Esta fase inicial incluía un gran número de preguntas distribuidas en tres partes: datos personales, enseñanza del inglés como L2 y herramientas tecnológicas. La segunda fase fue el proceso para la comprobación de las características del cuestionario

La modalidad semipresencial y la pronunciación de la lengua inglesa: Resultados de un modelo apoyado con TIC. Ana $\mathrm{M}^{\mathrm{a}}$ Pinto, $\mathrm{M}^{\mathrm{a}}$ Cruz Sánchez, Francisco J. García-Peñalvo y Marcos Cabezas. 
y la validez externa del instrumento. Se llevó a cabo una validación por interjueces. Se contó con cinco expertos de los cuales uno era experto en didáctica, dos en metodología, uno en inglés y uno en nuevas tecnologías. Atendieron principalmente al contenido de las preguntas, a la claridad y la redacción de estas, a la adecuación de los términos empleados, a la relevancia de los ítems con las dimensiones estudiadas y la ubicación de las diferentes preguntas en el cuestionario. Los expertos analizaron el cuestionario inicial y remitieron un análisis crítico, realizando aportaciones y sugerencias. En la tercera y última fase del diseño del cuestionario se realizó un análisis y estudio de las diferentes aportaciones de los expertos y sobre la base de las sugerencias y aportaciones de estos, se realizaron los cambios en el instrumento, el cual quedó estructurado en tres bloques: datos personales, tecnología e inglés como L2. Las preguntas de los dos últimos bloques se mezclaron para evitar que los estudiantes contestaran al azar. Finalmente el primer cuestionario tenía 21 ítems y el segundo 25.

Para prevenir posibles efectos negativos de la aplicación del cuestionario: efecto halo, tendencia respuesta, etc., se utilizaron preguntas de diferente tipología: abiertas, cerradas, respuesta múltiple y escala likert de 1 a 5 siguiendo estos criterios:

- $\quad 1=$ bajo, $2=$ regular, $3=$ bueno, $4=$ muy bueno, $5=$ excelente.

- $\quad 1=$ nunca, $2=$ casi nunca, $3=$ a veces, $4=$ casi siempre, $5=$ siempre.

- 1 = ineficaz, 2 = algo ineficaz, $3=$ neutral, $4=$ eficaz, $5=$ muy eficaz.

- 1 = totalmente en desacuerdo, 2 = en desacuerdo, $3=$ ni de acuerdo ni en desacuerdo, $4=$ de acuerdo $5=$ totalmente de acuerdo.

Para comprobar la consistencia interna de los cuestionarios, se utilizó el índice de consistencia interna, $\alpha$ de Cronbach. El coeficiente alfa del primer cuestionario tuvo un valor de 0,819 y el segundo de 0,824. Como Thorndike (1997) señala el conseguir un valor de 0,6 en el coeficiente alfa, es entendido como un valor aceptable. El valor obtenido en los dos cuestionarios utilizados en nuestra investigación ha superado ampliamente este valor, por lo que podemos decir que nuestros cuestionarios tienen una alta consistencia interna y alta fiabilidad, por lo que sus mediciones son estables y consistentes.

\section{Trabajo de campo}

Los cuestionarios fueron completados por los participantes en la investigación durante la primera y la última sesión presencial de la asignatura de Idioma I (inglés). Una vez terminado el trabajo de campo se procedió al ordenamiento de los datos obtenidos. En primer lugar, se preparó el registro de codificación para posteriormente procesar los 716 cuestionarios recogidos en un fichero ad hoc. Después de crear la plantilla o matriz, se introdujeron los datos, se depuraron y realización los análisis de carácter descriptivo e inferencial con la ayuda del programa estadístico IBM SPSS 20.

Realizamos, en primer lugar, un análisis descriptivo de las variables de los dos cuestionarios, expresados en frecuencias y porcentajes; y, en segundo lugar, un análisis

\footnotetext{
La modalidad semipresencial y la pronunciación de la lengua inglesa: Resultados de un modelo apoyado con TIC. Ana $\mathrm{M}^{\mathrm{a}}$ Pinto, $\mathrm{M}^{\mathrm{a}}$ Cruz Sánchez, Francisco J. García-Peñalvo y Marcos Cabezas. 
inferencial, realizando la prueba de significación de diferencia de medias en muestras dependientes (prueba t-student) y el análisis de varianza ANOVA de un factor.

Los resultados cuantitativos obtenidos se han ilustrado con figuras y tablas en Microsoft Office Word 2010.

\section{Resultados}

Presentamos los principales resultados del análisis descriptivo e inferencial realizado en los datos obtenidos en relación con los diferentes grupos de edad.

\section{Pronunciación de la lengua inglesa}

En consonancia con las expectativas, el análisis de datos reveló que la mayoría de los estudiantes $(66,8 \%)$ tuvieron la percepción de haber mejorado bastante su pronunciación de la lengua inglesa a través de las herramientas tecnológicas utilizadas en el modelo implementado.

Analizamos si existían diferencias estadísticamente significativas (IC 95\%) entre los diferentes grupos de edad que constituían la muestra en la variable dependiente sobre la pronunciación del inglés y obtuvimos que sí existían dichas diferencias estadísticamente significativas entre los diferentes grupos $(\mathrm{p}=, 002)$. Calculamos la prueba post-hoc de Tukey (Tabla 1) que reveló las diferencias que existían entre los estudiantes más jóvenes con edades comprendidas entre los 20 y los 24 años y los dos grupos de mayor edad, de 30 a 34, y de 35 o más de 35 años. De igual forma obtuvimos que existían diferencias estadísticamente significativas entre los estudiantes del grupo de edad de 25 a 29 años y los de 35 o más de 35 años. Sin embargo, no existían tales diferencias entre los estudiantes que formaban parte de los grupos de edades más jóvenes de 20 a 24 y de 25 a 29, y entre los participantes con edades comprendidas entre los de 25-29 y los de 30-34 años.

Los estudiantes valoraron su pronunciación de la lengua inglesa en una escala de 0 a 10 . Los dos grupos de participantes de menor edad (20-24 y 25-29 años) valoraron mejor su nivel ( $\bar{x}=7,23$ y $\bar{x}=7,20)$ que los dos grupos formados por sujetos de mayor edad (30-34 $\overline{\mathrm{X}}=6,87$ y 35 o más de 35 años $\overline{\mathrm{x}}=6,73$ )

Tabla 1. Comparaciones múltiples

Variable dependiente: Nivel de pronunciación lengua inglesa

\begin{tabular}{lllcl}
\hline & $\begin{array}{c}\text { (I) Edad } \\
\text { categorizada }\end{array}$ & \multicolumn{1}{c}{$\begin{array}{c}\text { (J) Edad } \\
\text { categorizada }\end{array}$} & $\begin{array}{c}\text { Diferencia de } \\
\text { medias (I-J) }\end{array}$ & Sig. \\
\hline HSD de & $20-24$ & $30-34$ & $0,359^{*}$ & 0,041 \\
Tukey & & 35 o más & $0,503^{*}$ & 0,014 \\
\cline { 3 - 5 } & $25-29$ & 35 o más & $0,468^{*}$ & 0,024 \\
\hline
\end{tabular}

*La diferencia de medias es significativa al nivel $0,05 . \mathrm{n}=358$

La modalidad semipresencial y la pronunciación de la lengua inglesa: Resultados de un modelo apoyado con TIC. Ana $\mathrm{M}^{\mathrm{a}}$ Pinto, $\mathrm{M}^{\mathrm{a}}$ Cruz Sánchez, Francisco J. García-Peñalvo y Marcos Cabezas. 
De igual forma analizamos si existían diferencias estadísticamente significativas (IC 95\%) entre esta variable dependiente en el cuestionario inicial y el final y se apreció que existía diferencia (Tabla 2) en la media del nivel de la pronunciación de la lengua inglesa $(\mathrm{t}=-23,581)$. Por lo tanto se rechaza la hipótesis nula $(\mathrm{H} 1)$ e indicamos que existe una relación entre la mejora de la pronunciación de la lengua inglesa y las herramientas propuestas en el modelo que permiten que esta mejore.

Tabla 2. Correlaciones de muestras relacionadas

\begin{tabular}{lccc}
\hline & N & Correlación & Sig. \\
\hline $\begin{array}{c}\text { Par 1 Pronunciación lengua inglesa - } \\
\text { Pronunciación lengua inglesa }\end{array}$ & 358 & 0,172 & 0,001 \\
\hline *95\% Intervalo de confianza para la diferencia. $\mathrm{n}=358$ & & \\
\end{tabular}

Frecuencia de uso de podcast, videocast, cuestionarios online, glosario online y foros al comienzo y al final del curso

En los resultados obtenidos en las variables dependientes que hacían referencia a la frecuencia de uso de las distintas herramientas tecnológicas utilizadas en el modelo (Podcast, Videocast, cuestionarios online, glosario online y foros) antes de comenzar la asignatura de Idioma I (inglés) destacamos que los mayores porcentajes obtenidos en podcast y videocast fluctúan entre los que no los han utilizado nunca $(34,4 \%$ y $37,7 \%$ respectivamente) y los que los utilizan casi siempre (37,2\% y 34,6\% respectivamente), solamente el 10,9 y el 8,7\% respectivamente aseguran utilizarlos siempre. En relación a la frecuencia de uso de los cuestionarios online casi la totalidad de los sujetos señalaron que no los utilizaban nunca $(50,8 \%)$ o casi nunca $(32,1 \%)$. En el caso de la frecuencia de uso del glosario online los mayores porcentajes se obtuvieron en las valoraciones que hacían referencia a nunca $(22,9 \%)$, casi nunca $(31,8 \%)$ y a veces $(29,1 \%)$. Finalmente, destacamos que el 25,4\% señaló que casi nunca utilizaba los foros, el 22,3\% que los usaba a veces y el $23,5 \%$ que los utilizaba casi siempre.

Analizamos si existían diferencias estadísticamente significativas (IC 95\%) entre los diferentes grupos de edad y las variables que se referían a los Podcast, Videocast, cuestionarios online, glosario online y foros. Podemos señalar la existencia de diferencias entre los distintos grupos de edad en las variables dependientes que se referían a la frecuencia de uso (podcast $\mathrm{p}=, 000$, videocast $\mathrm{p}=, 000$, cuestionarios online $\mathrm{p}=, 000$, glosarios online $\mathrm{p}=, 000 \mathrm{y}$ foros $\mathrm{p}=, 000$ ) al comienzo del curso. A partir de los resultados obtenidos, podemos apreciar que existen diferencias significativas en la media de la frecuencia de uso de cualquiera de estos recursos entre el conjunto de los grupos.

Se calculó la prueba post-hoc de Tukey que reveló que existían diferencias estadísticamente significativas entre los estudiantes de los dos grupos de edades más jóvenes (20-24 y 25-29) y los grupos de edades comprendidas entre los 30-34 y entre 35 o más de 35 años en la frecuencia de uso de podcast, videocast, cuestionarios online, glosario online y foros (Tabla 3). Sin embargo, no existieron diferencias significativas

La modalidad semipresencial y la pronunciación de la lengua inglesa: Resultados de un modelo apoyado con TIC. Ana $\mathrm{M}^{\mathrm{a}}$ Pinto, $\mathrm{M}^{\mathrm{a}}$ Cruz Sánchez, Francisco J. García-Peñalvo y Marcos Cabezas. 
entre los estudiantes que formaban parte de los grupos de edades de 20 a 24 y de 25 a 29.

Los estudiantes de los grupos más jóvenes (20-24 y 25-29 años) valoraron que utilizaban con más frecuencia las herramientas tecnológicas indicadas (20-24 años: Podcast $\bar{x}=4,20$ videocast $\bar{x}=4,09$, cuestionarios online $\bar{x}=1,90$, glosario online $\bar{x}=3,21$ y foros $\bar{x}=4,25 ; 25-29$ años: Podcast $\bar{x}=3,90$ videocast $\bar{x}=3,83$, cuestionarios online $\bar{x}=1,87$, glosario online $\bar{x}=2,89$ y foros $\bar{x}=4,01$ ) que los dos grupos formados por sujetos de mayor edad (30-34 años: Podcast $\bar{x}=1,55$ videocast $\bar{x}=1,41$, cuestionarios online $\bar{x}=1,53$, glosario online $\bar{x}=1,77$ y foros $\bar{x}=2,10 ; 35$ o más de 35 años: Podcast $\bar{x}=1,45$ videocast $\bar{x}=1,33$, cuestionarios online $\bar{x}=1,40$, glosario online $\bar{x}=1,76$ y foros $\bar{x}=1,93$ ).

Tabla 3. Comparaciones múltiples

Variable dependiente: Frecuencia de uso de los Podcast

\begin{tabular}{|c|c|c|c|c|}
\hline & $\begin{array}{c}\text { (I) Edad } \\
\text { categorizada }\end{array}$ & $\begin{array}{c}(\mathrm{J}) \text { Edad } \\
\text { categorizada }\end{array}$ & Diferencia de medias (I-J) & Sig. \\
\hline HSD de & $20-24$ & $30-34$ & $2,647 *$ & ,000 \\
\hline \multirow[t]{3}{*}{ Tukey } & & 35 o más & $2,741 *$ &, 000 \\
\hline & $25-29$ & $30-34$ & $2,354 *$ & ,000 \\
\hline & & 35 o más & $2,448 *$ & ,000 \\
\hline \multicolumn{5}{|c|}{ Variable dependiente: Frecuencia de uso de los Videocast } \\
\hline HSD de & $20-24$ & $30-34$ & $2,681^{*}$ & ,000 \\
\hline \multirow[t]{3}{*}{ Tukey } & & 35 o más & $2,765^{*}$ &, 000 \\
\hline & $25-29$ & $30-34$ & $2,415^{*}$ & 000 \\
\hline & & 35 o más & $2,499 *$ &, 000 \\
\hline \multicolumn{5}{|c|}{ Variable dependiente: Frecuencia de uso de los Cuestionarios Online } \\
\hline HSD de & $20-24$ & $30-34$ & ,364* & ,006 \\
\hline \multirow[t]{3}{*}{ Tukey } & & 35 o más & ,497* & ,002 \\
\hline & $25-29$ & $30-34$ & $337 *$ & 011 \\
\hline & & 35 o más &, $470 *$ & ,003 \\
\hline \multicolumn{5}{|c|}{ Variable dependiente: Frecuencia de uso de los Glosario Online } \\
\hline HSD de & $20-24$ & $30-34$ & $1,433^{*}$ & 000 \\
\hline \multirow[t]{3}{*}{ Tukey } & & 35 o más & $1,443 *$ &, 000 \\
\hline & $25-29$ & $30-34$ & $1,117 *$ & 000 \\
\hline & & 35 o más & $1,128 *$ &, 000 \\
\hline \multicolumn{5}{|c|}{ Variable dependiente: Frecuencia de uso de los Foros } \\
\hline HSD de & $20-24$ & $30-34$ & $2,156^{*}$ & ,000 \\
\hline \multirow[t]{3}{*}{ Tukey } & & 35 o más & $2,326 *$ &, 000 \\
\hline & $25-29$ & $30-34$ & $1,914 *$ & ,000 \\
\hline & & 35 o más & $2,084 *$ & 000 \\
\hline
\end{tabular}

*La diferencia de medias es significativa al nivel $0,05 . \mathrm{n}=358$

En relación a los resultados obtenidos en las valoraciones finales que realizaron los estudiantes sobre la frecuencia de uso de las diferentes herramientas tecnológicas utilizadas en el modelo (Podcast, Videocast, cuestionarios online, glosario online y

La modalidad semipresencial y la pronunciación de la lengua inglesa: Resultados de un modelo apoyado con TIC. Ana $\mathrm{M}^{\mathrm{a}}$ Pinto, $\mathrm{M}^{\mathrm{a}}$ Cruz Sánchez, Francisco J. García-Peñalvo y Marcos Cabezas. 
foros) para el aprendizaje de la pronunciación de la lengua inglesa, destacamos que la mayoría de los estudiantes valoraron que habían utilizado los podcast, videocast, glosario online y foros con bastantes frecuencia, fluctuando la mayores valoraciones entre casi siempre $(40,8 \%, 45,5 \%, 54,5 \%$ y $39,9 \%$ respectivamente) y siempre $(32,1 \%$, $28,8 \%, 33,2 \%$ y $50 \%$ respectivamente). En el caso de los cuestionarios online destacamos que el $43 \%$ valoraron que los utilizaron casi siempre, el $26,5 \%$ a veces y el $29,3 \%$ siempre.

Analizamos si existían diferencias estadísticamente significativas (IC 95\%) entre los diferentes grupos de edad y las variables que se referían a los Podcast, Videocast, cuestionarios online, glosario online y foros. Realizados los análisis, podemos señalar que existían diferencias significativas entre los distintos grupos de edad en las variables dependientes que se referían a la frecuencia de uso de podcats $(\mathrm{p}=, 000)$, videocast $(\mathrm{p}=.000)$ y foros $(\mathrm{p}=.000)$ al finalizar el curso. A partir de los resultados obtenidos, apreciamos la existencia de diferencias estadísticamente significativas en la media de la frecuencia de uso de estos recursos entre el conjunto de los grupos de edad.

Procedimos al cálculo de la prueba post-hoc de Tukey que mostró la existencia de diferencias estadísticamente significativas entre los estudiantes de los dos grupos de edades más jóvenes (20-24 y 25-29) y los grupos de edades comprendidas entre los 3034 y entre 35 o más de 35 años en la frecuencia de uso de podcast, videocast y foros (Tabla 4). Sin embargo, no existieron diferencias significativas en estas tres variables entre los estudiantes que formaban parte de los grupos de edades de 20 a 24 y de 25 a 29.

Los sujetos más jóvenes (20-24 y 25-29 años) valoraron que utilizaban con más frecuencia las herramientas tecnológicas indicadas (20-24 años: Podcast $\bar{x}=4,54$ videocast $\bar{x}=4,41$ y foros $\bar{x}=4,69 ; 25-29$ años: Podcast $\bar{x}=4,32$ videocast $\bar{x}=4,22$ y foros $\bar{x}=4,61)$ que los dos grupos formados por sujetos de mayor edad (30-34 años: Podcast $\bar{x}=3,44$ videocast $\bar{x}=3,71$ y foros $\bar{x}=4,13 ; 35$ o más de 35 años: Podcast $\bar{x}=3,38$ videocast $\bar{x}=3,42$ y foros $\bar{x}=4,18$ ).

Tabla 4. Comparaciones múltiples

Variable dependiente: Frecuencia de uso de los Podcast

\begin{tabular}{|c|c|c|c|c|}
\hline & $\begin{array}{c}\text { (I) Edad } \\
\text { categorizada }\end{array}$ & $\begin{array}{c}\text { (J) Edad } \\
\text { categorizada }\end{array}$ & Diferencia de medias (I-J) & Sig. \\
\hline \multirow{4}{*}{$\begin{array}{l}\text { HSD de } \\
\text { Tukey }\end{array}$} & $20-24$ & $30-34$ & $1,105^{*}$ & ,000 \\
\hline & & 35 o más & $1,158 *$ & ,000 \\
\hline & $25-29$ & $30-34$ & ,880* & 000 \\
\hline & & 35 o más & ,933* & ,000 \\
\hline \multicolumn{5}{|c|}{ Variable dependiente: Frecuencia de uso de los Videocast } \\
\hline HSD de & $20-24$ & $30-34$ & ,704* & ,000 \\
\hline \multirow[t]{3}{*}{ Tukey } & & 35 o más & ,996* &, 000 \\
\hline & $25-29$ & $30-34$ & ,508* & 000 \\
\hline & & 35 o más & ,799* &, 000 \\
\hline
\end{tabular}

Variable dependiente: Frecuencia de uso de los Foros

La modalidad semipresencial y la pronunciación de la lengua inglesa: Resultados de un modelo apoyado con TIC. Ana $\mathrm{M}^{\mathrm{a}}$ Pinto, $\mathrm{M}^{\mathrm{a}}$ Cruz Sánchez, Francisco J. García-Peñalvo y Marcos Cabezas. 


\begin{tabular}{lllll}
\hline HSD de & $20-24$ & $30-34$ &, $561^{*}$ &, 000 \\
Tukey & & 35 o más &, $508^{*}$ &, 000 \\
\cline { 3 - 5 } & $25-29$ & $30-34$ &, $480^{*}$ &, 000 \\
& & 35 o más &, $427^{*}$ &, 000 \\
\hline
\end{tabular}

*La diferencia de medias es significativa al nivel 0,05. $\mathrm{n}=358$

De igual forma analizamos si existían diferencias estadísticamente significativas (IC 95\%) entre las variables dependientes sobre la frecuencia de uso de las diferentes herramientas tecnológicas (podcast, videocast, cuestionarios online, glosario online y foros) en el cuestionario inicial y el final y se apreció que existía diferencia (Tabla 5) en la media de frecuencia de uso de podcast $(\mathrm{t}=-15,280)$, videocast $(\mathrm{t}=-17,285)$ y foros $(\mathrm{t}=-19,996)$. Por lo tanto se rechaza la hipótesis nula $(\mathrm{H} 2)$ e indicamos que existe una relación entre el aumento del uso de estas herramientas para el aprendizaje de la pronunciación de la lengua inglesa y el modelo tecnológico propuesto que favorece su uso.

Tabla 5. Correlaciones de muestras relacionadas

\begin{tabular}{lccc}
\hline & $\mathrm{N}$ & Correlación & Sig. \\
\hline $\begin{array}{l}\text { Par 1 Frecuencia de uso de podcast - } \\
\text { Frecuencia de uso de podcast }\end{array}$ & 358 &, 426 &, 000 \\
\hline $\begin{array}{l}\text { Par 2 Frecuencia de uso de videocast - } \\
\text { Frecuencia de uso de videocast }\end{array}$ & 358 &, 361 &, 000 \\
\hline $\begin{array}{l}\text { Par 3 Frecuencia de uso de cuestionarios } \\
\text { online - Frecuencia de uso de cuestionarios } \\
\text { online }\end{array}$ & 358 &, 020 &, 700 \\
\hline $\begin{array}{l}\text { Par 4 Frecuencia de uso de glosario online - } \\
\text { Frecuencia de uso de glosario online }\end{array}$ & 358 &,- 016 &, 764 \\
\hline $\begin{array}{l}\text { Par 5 Frecuencia de uso de foros - } \\
\text { Frecuencia de uso de foros }\end{array}$ & 358 &, 321 &, 000 \\
\hline *95\% Intervalo de confianza para la diferencia. n $=358$ & &
\end{tabular}

\section{Percepciones de los estudiantes sobre la eficacia de podcast, videocast, cuestionarios online, glosario online y foros}

Nuestros resultados indican que la mayoría de los estudiantes valoran la eficacia de los podcast y los videocast entre eficaz $(38,3 \%$ y $37,7 \%$ respectivamente) y muy eficaz (45\% y 48,9\% respectivamente), solamente el $2,2 \%$ en el caso de los podcast y un $0,8 \%$ en su valoración de los videocast consideran que el uso que se ha realizado de estas herramientas es algo ineficaz. En relación al uso de los cuestionarios online, los glosarios online y los foros, la mayoría de las valoraciones fluctuaron entre eficaces $(36,6 \%, 38,5 \%$ y $44,4 \%$ respectivamente) y muy eficaces $(32,7 \%, 33,8 \%$ y $38,3 \%$ respectivamente). Ningún estudiante las valoró como ineficaces.

Analizamos si existían diferencias estadísticamente significativas (IC 95\%) entre los diferentes grupos de edad en las variables dependientes que se referían a la eficacia de las herramientas transmisivas, interactivas y colaborativas usadas en el modelo

La modalidad semipresencial y la pronunciación de la lengua inglesa: Resultados de un modelo apoyado con TIC. Ana $\mathrm{M}^{\mathrm{a}}$ Pinto, $\mathrm{M}^{\mathrm{a}}$ Cruz Sánchez, Francisco J. García-Peñalvo y Marcos Cabezas. 
tecnológico implementado. Indicamos la existencia de diferencias entre los grupos en dichas variables podcast $(p=, 000)$, videocast $(p=, 000)$, cuestionarios online $(p=, 000)$, glosario online $(\mathrm{p}=, 000)$ y foros $(\mathrm{p}=, 000)$. A partir de los resultados obtenidos, podemos apreciar que existen diferencias significativas en la media de la eficacia de uso de estos recursos entre el conjunto de los grupos.

Los resultados obtenidos al calcular la prueba post-hoc de Tukey indicaron que existían diferencias estadísticamente significativas entre los sujetos de los dos grupos de edades más jóvenes (20-24 y 25-29) y los grupos de edades comprendidas entre los 30-34 y entre 35 o más de 35 años en la eficacia de uso de podcast, videocast, cuestionarios online, glosario online y foros (Tabla 6). Sin embargo, los análisis demostraron que dichas diferencias no existían en el caso de los estudiantes de los grupos de edades más jóvenes (20-24 y 25-29).

Los dos grupos de alumnos más jóvenes (20-24 y 25-29) evaluaron mejor la eficacia de estas herramientas (Podcast: $20-24, \bar{x}=4,67$ y 25-29, $\bar{x}=4,58$; Videocast: 20-24, $\bar{x}=4,77$ y 25-29, $\bar{x}=4,58$; cuestionarios online: $20-24, \bar{x}=4,73$ y $25-29 ; \bar{x}=4,49$, glosario online: 20-24, $\bar{x}=4,67$ y 25-29, $\bar{x}=4,63$ y foros: $20-24, \bar{x}=4,63$ y 25-29, $\bar{x}=4,50$ ) que los grupos de mayor edad (Podcast: $30-34, \bar{x}=3,95$ y 35 o más de $35, \bar{x}=3,78$, Videocast: $30-34$, $\bar{x}=4,09$ y 35 o más de $35, \bar{x}=3,84$, cuestionarios online: $30-34, \bar{x}=3,10$ y 35 o más de $35, \bar{x}=3,35$, glosario online: $30-34, \bar{x}=3,40$ y 35 o más de $35, \bar{x}=3,38$ y foros: $30-34$, $\overline{\mathrm{X}}=3,77$ y 35 o más de $35, \bar{x}=3,96$ ).

Tabla 6. Comparaciones múltiples

Variable dependiente: Percepciones de los estudiantes sobre la eficacia de los podcast

\begin{tabular}{|c|c|c|c|c|}
\hline & $\begin{array}{c}\text { (I) Edad } \\
\text { categorizada }\end{array}$ & $\begin{array}{l}\text { (J) Edad } \\
\text { categorizada }\end{array}$ & Diferencia de medias (I-J) & Sig. \\
\hline HSD de & $20-24$ & $30-34$ & ,715* & 000 \\
\hline \multirow[t]{3}{*}{ Tukey } & & 35 o más &, $885^{*}$ &, 000 \\
\hline & $25-29$ & $30-34$ & ,624* &, 000 \\
\hline & & 35 o más &, $794 *$ &, 000 \\
\hline \multicolumn{5}{|c|}{$\begin{array}{l}\text { Variable dependiente: Percepciones de los estudiantes sobre la eficacia de los } \\
\text { videocast }\end{array}$} \\
\hline HSD de & $20-24$ & $30-34$ & ,704* & ,000 \\
\hline \multirow[t]{3}{*}{ Tukey } & & 35 o más & ,957* &, 000 \\
\hline & $25-29$ & $30-34$ & $487 *$ & 000 \\
\hline & & 35 o más &, $740 *$ &, 000 \\
\hline \multicolumn{5}{|c|}{$\begin{array}{l}\text { Variable dependiente: Percepciones de los estudiantes sobre la eficacia de los } \\
\text { cuestionarios online }\end{array}$} \\
\hline HSD de & $20-24$ & $30-34$ & $1,650^{*}$ &, 000 \\
\hline \multirow[t]{3}{*}{ Tukey } & & 35 o más & $1,402 *$ &, 000 \\
\hline & $25-29$ & $30-34$ & $1,414^{*}$ & 000 \\
\hline & & 35 o más & $1,165^{*}$ &, 000 \\
\hline
\end{tabular}

La modalidad semipresencial y la pronunciación de la lengua inglesa: Resultados de un modelo apoyado con TIC. Ana $\mathrm{M}^{\mathrm{a}}$ Pinto, $\mathrm{M}^{\mathrm{a}}$ Cruz Sánchez, Francisco J. García-Peñalvo y Marcos Cabezas. 
Variable dependiente: Percepciones de los estudiantes sobre la eficacia del glosario online

\begin{tabular}{lcllr}
\hline HSD de & $20-24$ & $30-34$ & $1,286^{*}$ &, 000 \\
Tukey & $25-29$ & 35 o más & $1,308^{*}$ &, 000 \\
\cline { 3 - 5 } & & $30-34$ & $1,249^{*}$ &, 000 \\
& & 35 o más & $1,270^{*}$ &, 000 \\
\hline \multicolumn{4}{l}{ Variable dependiente: } & Percepciones de los estudiantes sobre la eficacia de los foros \\
\hline HSD de & $20-24$ & $30-34$ &, $858^{*}$ &, 000 \\
Tukey & & 35 o más &, $669^{*}$ &, 000 \\
\cline { 3 - 5 } & $25-29$ & $30-34$ &, $726^{*}$ &, 000 \\
& & 35 o más &, $536^{*}$ &, 000 \\
\hline
\end{tabular}

*La diferencia de medias es significativa al nivel $0,05 . \mathrm{n}=358$

Percepciones de los estudiantes sobre la eficacia de las e-actividades para desarrollar la competencia fonológica de los estudiantes, mejorar la pronunciación y las destrezas orales

En los resultados obtenidos en las valoraciones que los estudiantes realizan sobre la eficacia de las e-actividades para la mejora de la competencia fonológica, la pronunciación de la lengua inglesa y la mejora de las destrezas orales destacamos que la mayoría de los sujetos estaban de acuerdo con su eficacia $(47,8 \%, 67,9 \%$ y $71,2 \%$ respectivamente).

Señalamos la existencia de diferencias significativas en la eficacia de las e-actividades para desarrollar la competencia fonológica de los estudiantes, mejorar la pronunciación de la lengua inglesa y las destrezas orales (Tabla 7), entre los diferentes grupos de edad $(\mathrm{p}=, 000, \mathrm{p}=, 000$ y $\mathrm{p}=, 000)$. Estas diferencias existían en las tres variables entre los estudiantes más jóvenes (20-24 y 25-29) y los que conformaban los grupos de edades comprendidas entre los 30-34 y los de 35 o más de 35 años. Sin embargo, no se apreciaban en los resultados obtenidos en los dos grupos de estudiantes más jóvenes (20-24 y 25-29).

Los sujetos más jóvenes coincidieron en valorar de forma más efectiva las e-actividades desarrolladas en el modelo tecnológico para mejorar la competencia fonológica, la pronunciación y las destrezas orales (20-24: $\bar{x}=4,18, \bar{x}=4,06, \bar{x}=4,15$ y 25-29: $\bar{x}=3,95$, $\bar{x}=3,96, \bar{x}=4,15)$ que los estudiantes de los grupos de mayor edad (30-34: $\bar{x}=3,52$, $\bar{x}=3,61, \bar{x}=3,63$ y 35 o más de $35: \bar{x}=3,25, \bar{x}=3,36, \bar{x}=3,40$ ).

Tabla 7. Comparaciones múltiples

Variable dependiente: Gracias a las e-actividades, he desarrollado la competencia fonológica

\begin{tabular}{lllll}
\hline & \multicolumn{1}{c}{$\begin{array}{c}\text { (I) Edad } \\
\text { categorizada }\end{array}$} & \multicolumn{1}{c}{$\begin{array}{c}\text { (J) Edad } \\
\text { categorizada }\end{array}$} & Diferencia de medias (I-J) & Sig. \\
\hline $\begin{array}{l}\text { HSD de } \\
\text { Tukey }\end{array}$ & $20-24$ & $30-34$ &, $668^{*}$ &, 000 \\
& & 35 o más &, $929^{*}$ &, 001 \\
\hline
\end{tabular}

La modalidad semipresencial y la pronunciación de la lengua inglesa: Resultados de un modelo apoyado con TIC. Ana $\mathrm{M}^{\mathrm{a}}$ Pinto, $\mathrm{M}^{\mathrm{a}}$ Cruz Sánchez, Francisco J. García-Peñalvo y Marcos Cabezas. 


\begin{tabular}{|c|c|c|c|c|}
\hline & $25-29$ & $\begin{array}{l}\text { 30-34 } \\
35 \text { o más }\end{array}$ & $\begin{array}{l}, 430^{*} \\
, 691 *\end{array}$ & $\begin{array}{l}, 003 \\
, 000\end{array}$ \\
\hline Variable & endient & a las e-act & ado la & ión \\
\hline HSD de & $20-24$ & $30-34$ &, $445^{*}$ & ,000 \\
\hline Tukey & & 35 o más &, $694 *$ & ,000 \\
\hline & $25-29$ & $30-34$ & ,344* & ,001 \\
\hline & & 35 o más &, $593 *$ & ,000 \\
\hline $\begin{array}{l}\text { Variable } \\
\text { orales }\end{array}$ & idien & $s$ a las e- & $\overline{\text { ejorad }}$ & rezas \\
\hline HSD de & $20-24$ & $30-34$ &, $520 *$ & ,000 \\
\hline Tukey & & 35 o más &, $749 *$ & ,000 \\
\hline & $25-29$ & $30-34$ & ,404* & ,000 \\
\hline & & 35 o más &, $633 *$ & ,000 \\
\hline
\end{tabular}

*La diferencia de medias es significativa al nivel $0,05 . \mathrm{n}=358$

En vista a los resultados obtenidos la mayoría de los estudiantes afirma que las eactividades desarrolladas han sido muy útiles para mejorar su pronunciación del inglés. El 44,4\% está de acuerdo y el 49,2\% totalmente de acuerdo, solamente un 1,1\% señala su desacuerdo con dicha afirmación.

\section{EI EVA como entorno para ayudar a los estudiantes a desarrollar su habilidad para percibir y producir la lengua inglesa con mayor precisión}

La mayoría de participantes en la investigación valoraron entre de acuerdo $(42,2 \%)$ y totalmente de acuerdo (41,3\%) el EVA como un entorno que favorece el desarrollo de la habilidad de los estudiantes para percibir y producir el inglés con mayor precisión. En la Tabla 8 se puede observar que en los resultados obtenidos existían diferencias estadísticamente significativas en las valoraciones entre los diferentes grupos de edad en esta variable. Dichas diferencias existían entre los estudiantes más mayores (35 o más de 35 años) y el resto de los grupos de edades comprendidas entre los 20-24 ( $\mathrm{p}=, 000)$, $25-29(\mathrm{p}=, 001)$ y $30-34(\mathrm{p}=, 001)$.

La mayoría de los grupos de edad consideraron que el EVA implementado les había proporcionado las herramientas y e-actividades adecuadas para desarrollar su habilidad para percibir y producir el inglés con mayor precisión (20-24: $\bar{x}=4,38,25-29: \bar{x}=4,24$ y 30-34: $\bar{x}=4,19)$. Por otro lado, si tenemos en cuenta las opiniones del grupo de edad más mayor (35 o más de 35 años), apreciamos que la mayoría ni está de acuerdo ni en desacuerdo $(\overline{\mathrm{X}}=3,63)$.

Tabla 8. Comparaciones múltiples

Variable dependiente: El EVA implementado me ha ayudado a desarrollar la habilidad de percibir y producir la lengua inglesa de forma más precisa

\begin{tabular}{cccc}
$\begin{array}{c}\text { (I) Edad } \\
\text { categorizada }\end{array}$ & $\begin{array}{c}\text { (J) Edad } \\
\text { categorizada }\end{array}$ & $\begin{array}{c}\text { Diferencia de } \\
\text { medias (I-J) }\end{array}$ & Sig. \\
\hline
\end{tabular}

La modalidad semipresencial y la pronunciación de la lengua inglesa: Resultados de un modelo apoyado con TIC. Ana $\mathrm{M}^{\mathrm{a}}$ Pinto, $\mathrm{M}^{\mathrm{a}}$ Cruz Sánchez, Francisco J. García-Peñalvo y Marcos Cabezas. 


\begin{tabular}{lllll}
\hline HSD de & $20-24$ & 35 o más & $0,743^{*}$ & 0,000 \\
\cline { 2 - 5 } Tukey & $25-29$ & 35 o más & $0,603^{*}$ & 0,001 \\
\cline { 2 - 5 } & $30-34$ & 35 o más & $0,549^{*}$ & 0,001 \\
\hline
\end{tabular}

*La diferencia de medias es significativa al nivel 0,05. $\mathrm{n}=358$

Más de la mitad de los estudiantes $(69,6 \%)$ señalan que están de acuerdo con la eficacia de las e-actividades para ser más conscientes de la pronunciación del inglés, así como de los diferentes acentos. Un mínimo porcentaje $(1,7 \%)$ muestra su total desacuerdo con esta afirmación.

Consideran que el EVA implementado les ha proporcionado un entorno natural para la práctica de la pronunciación de la lengua inglesa. El 45,5\% está de acuerdo y el $45 \%$ totalmente de acuerdo. Un mínimo porcentaje $(0,6 \%$ y $2,2 \%)$ considera que está en desacuerdo o totalmente en desacuerdo con esta afirmación.

\section{Autoevaluación de los estudiantes de su nivel de las destrezas orales y la pronunciación de la lengua inglesa}

Los resultados obtenidos muestran que la mayoría de los estudiantes al finalizar el curso han valorado entre buena y muy buena la mejora de su nivel tanto de las destrezas orales como de la pronunciación del inglés. En el caso de la comprensión oral el 27,9\% de los participantes valoraron su nivel como bueno y el 58,4\% como muy bueno. Si nos detenemos en los resultados de la valoración del nivel de la expresión oral los mayores porcentajes los obtenemos en un nivel bueno $41,1 \%$ y muy bueno $46,1 \%$. Finalmente, destacamos que la valoración del nivel de la pronunciación de la lengua inglesa es aún mejor y los participantes la califican entre bueno $21,2 \%$, muy bueno $54,2 \%$ y excelente $21,8 \%$.

Destacamos la existencia de diferencias estadísticamente significativas en la autoevaluación de los estudiantes de las variables dependientes: comprensión oral, expresión oral y pronunciación de la lengua inglesa (Tabla 9) entre los diferentes grupos de edad ( $\mathrm{p}=.000, \mathrm{p}=.000 \mathrm{y} \mathrm{p}=.000$ ). El cálculo de la prueba post-hoc de Tukey destacó la existencia de estas diferencias en las tres variables entre los estudiantes más jóvenes (20-24 y 25-29) y los que conformaban los grupos de edades comprendidas entre los 3034 y los de 35 o más de 35 años. Sin embargo, no se apreciaban dichas diferencias en los resultados obtenidos en los dos grupos de estudiantes más jóvenes (20-24 y 25-29).

Los resultados muestran como los sujetos que conformaban los grupos de estudiantes más jóvenes (20-24 y 25-29 años) valoraron mejor su evolución en las destrezas orales y en la pronunciación de la lengua inglesa (20-24 años: comprensión oral $\bar{x}=4,05$, expresión oral $\bar{x}=3,77$ y pronunciación de la lengua inglesa $\bar{x}=4,24 ; 25-29$ años: comprensión oral $\bar{x}=3,92$, expresión oral $\bar{x}=3,71$ y pronunciación de la lengua inglesa $\overline{\mathrm{X}}=4,12$ ) que los dos grupos formados por sujetos de mayor edad (30-34 años: comprensión oral $\bar{x}=3,40$, expresión oral $\bar{x}=3,02$ y pronunciación de la lengua inglesa

\footnotetext{
La modalidad semipresencial y la pronunciación de la lengua inglesa: Resultados de un modelo apoyado con TIC. Ana $\mathrm{M}^{\mathrm{a}}$ Pinto, $\mathrm{M}^{\mathrm{a}}$ Cruz Sánchez, Francisco J. García-Peñalvo y Marcos Cabezas. 
$\bar{x}=3,77 ; 35$ o más de 35 años: comprensión oral $\bar{x}=3,24$, expresión oral $\bar{x}=2,96$ y pronunciación de la lengua inglesa $\bar{x}=3,58$ ).

Tabla 9. Comparaciones múltiples

Variable dependiente: Autoevaluación de los estudiantes de la comprensión oral (listening)

\begin{tabular}{|c|c|c|c|c|}
\hline & $\begin{array}{c}\text { (I) Edad } \\
\text { categorizada }\end{array}$ & $\begin{array}{c}\text { (J) Edad } \\
\text { categorizada }\end{array}$ & $\begin{array}{l}\text { Diferencia de medias } \\
(\mathrm{I}-\mathrm{J})\end{array}$ & Sig. \\
\hline HSD de & $20-24$ & $30-34$ & $0,651^{*}$ & 0,000 \\
\hline \multirow[t]{3}{*}{ Tukey } & & 35 o más & $0,810 *$ & 0,000 \\
\hline & $25-29$ & $30-34$ & $0,529 *$ & 0,000 \\
\hline & & 35 o más & $0,688 *$ & 0,000 \\
\hline \multicolumn{5}{|c|}{$\begin{array}{l}\text { Variable dependiente: Autoevaluación de los estudiantes de la expresión oral } \\
\text { (speaking) }\end{array}$} \\
\hline HSD de & $20-24$ & $30-34$ & $0,754 *$ & 0,000 \\
\hline \multirow[t]{3}{*}{ Tukey } & & 35 o más & $0,806^{*}$ & 0,000 \\
\hline & $25-29$ & $30-34$ & $0,690 *$ & 0,000 \\
\hline & & 35 o más & $0,743 *$ & 0,000 \\
\hline \multicolumn{5}{|c|}{$\begin{array}{l}\text { Variable dependiente: Autoevaluación de los estudiantes de su nivel de } \\
\text { pronunciación de la lengua inglesa }\end{array}$} \\
\hline HSD de & $20-24$ & $30-34$ & $0,475^{*}$ & 0,000 \\
\hline \multirow[t]{3}{*}{ Tukey } & & 35 o más & $0,660 *$ & 0,000 \\
\hline & $25-29$ & $30-34$ & $0,375^{*}$ & 0,001 \\
\hline & & 35 o más & $0,559 *$ & 0,000 \\
\hline
\end{tabular}

*La diferencia de medias es significativa al nivel 0,05. $\mathrm{n}=358$

Analizamos si existían diferencias estadísticamente significativas (IC 95\%) entre los ítems del primer (pre-test) y del último cuestionario (post-test) que se referían a la autoevaluación que realizaban los estudiantes de sus destrezas orales (listening y speaking) y pronunciación de la lengua inglesa, encontrando diferencias (Tabla 10) en la media de la expresión oral (speaking, $\mathrm{t}=-22.578$ ), comprensión oral (listening, $\mathrm{t}=$ 21.313) y de la pronunciación de la lengua inglesa $(\mathrm{t}=-24.036)$. Por lo tanto, se rechaza la hipótesis nula $(\mathrm{H} 3)$ e indicamos que existe una relación entre la mejora del nivel de los estudiantes de sus destrezas orales y de la pronunciación de la lengua inglesa, y la eficacia de las actividades propuestas en el modelo implementado en la asignatura de Idioma I (inglés). Los participantes en el estudio percibieron que mejoraron claramente su nivel en estos tres aspectos de la lengua inglesa (expresión oral, comprensión oral y pronunciación).

Tabla 10: Correlaciones de muestras relacionadas

\begin{tabular}{llcc}
\hline & N & Correlación & Sig. \\
\hline Par 1 Autoevaluación del estudiante de su expresión & 87 & 0,197 & 0,000 \\
$\begin{array}{l}\text { oral de la lengua inglesa - Autoevaluación del } \\
\text { estudiante de su expresión oral de la lengua } \\
\text { inglesa }\end{array}$ & & & \\
\hline
\end{tabular}

La modalidad semipresencial y la pronunciación de la lengua inglesa: Resultados de un modelo apoyado con TIC. Ana $\mathrm{M}^{\mathrm{a}}$ Pinto, $\mathrm{M}^{\mathrm{a}}$ Cruz Sánchez, Francisco J. García-Peñalvo y Marcos Cabezas. 


\begin{tabular}{lllll}
\hline Par $2 \begin{array}{l}\text { Autoevaluación del estudiante de su comprensión } \\
\text { oral de la lengua inglesa - Autoevaluación del } \\
\text { estudiante de su comprensión oral de la lengua } \\
\text { inglesa }\end{array}$ & & 0,155 & 0,003 \\
\hline $\begin{array}{l}\text { Par } 3 \begin{array}{l}\text { Autoevaluación del estudiante de su } \\
\text { pronunciación de la lengua inglesa - } \\
\text { Autoevaluación del estudiante de su } \\
\text { pronunciación de la lengua inglesa }\end{array} \\
\text { *95\% Intervalo de confianza para la diferencia. } \mathrm{n}=358\end{array}$ & & & \\
\hline
\end{tabular}

\section{Discusión de los resultados}

La finalidad de nuestro estudio fue analizar las percepciones de los estudiantes en relación con la eficacia para la pronunciación de la lengua inglesa de las diferentes herramientas transmisivas, interactivas y colaborativas utilizadas en el modelo tecnológico implementado.

De acuerdo a los resultados podemos afirmar que mejoró la percepción de los alumnos de la asignatura Idioma I (inglés) sobre su nivel de pronunciación de la lengua inglesa. Aspecto que se evidenció en los resultados en las correlaciones de muestras relacionadas en las variables dependientes que se referían a la pronunciación de la lengua inglesa en el pre-test y en el post-test. Además, también se puso de manifiesto que fueron los más jóvenes los que valoraron mejor o apreciaron más el potencial que cada una de las herramientas les brindaba. Los buenos resultados obtenidos en la eficacia de las herramientas tecnológicas van en línea con las conclusiones de los estudios llevados a cabo por Abdous, Camarena y Facer (2009) y por Pinto, Sánchez y García (2015) o en la investigación abordada por Chan (2012).

Los resultados del estudio apoyan la hipótesis de que las herramientas tecnológicas usadas, en concreto el uso de los podcast y videocast, facilitan que el discente tenga acceso a la práctica necesaria con un amplio rango de acentos y contextos para que mejore tanto la pronunciación de la lengua inglesa como las destrezas orales, y con ello sea capaz de comunicarse de una forma más eficaz en las distintas situaciones comunicativas, consiguiendo un aprendizaje significativo de la pronunciación de la lengua inglesa.

Se produjo una instrucción más individualizada, centrada en los discentes, en gran medida por las actividades que se presentaron en el modelo, en las que los alumnos se convirtieron en estudiantes activos, generando conocimiento (Pinto, Sánchez y García, 2015). Esto quedó evidenciado en la creación por parte de los discentes de los podcasts que se incluyeron en el modelo (Tallei y Coura, 2011). El hecho de ser participantes activos y proporcionarles oportunidades para tomar decisiones de manera individual a la hora de determinar ciertas actividades dentro del modelo, hizo que tuvieran una mayor motivación intrínseca a la hora de mejorar sus destrezas orales y su pronunciación del inglés (Man-Man, 2006).

\footnotetext{
La modalidad semipresencial y la pronunciación de la lengua inglesa: Resultados de un modelo apoyado con TIC. Ana $\mathrm{M}^{\mathrm{a}}$ Pinto, $\mathrm{M}^{\mathrm{a}}$ Cruz Sánchez, Francisco J. García-Peñalvo y Marcos Cabezas. 
La batería de pruebas presentadas en el Entorno virtual de aprendizaje (EVA), en concreto algunos de los cuestionarios online habilitados en distintos temas del modelo, facilitó que los estudiantes pudieran autoevaluar de forma sistemática su pronunciación de la lengua inglesa, su comprensión oral y su expresión oral (Pinto, Sánchez y García, 2016) y de esta forma conocer su evolución y necesidades.

Se hace evidente el sentido pedagógico y aplicado de las herramientas transmisivas, interactivas y colaborativas usadas en el modelo en el contexto de la enseñanza de la pronunciación de la lengua inglesa y la mejora de las destrezas orales. Son varios los estudios que destacan que estas herramientas son adecuadas para el aprendizaje en la modalidad semipresencial (Blended learning), ya que facilitan la práctica necesaria a los estudiantes de esta modalidad en la que el número de sesiones es más reducida que en la modalidad presencial (Pinto, Sánchez, Palacios, 2014; Ruiz, 2008). La tecnología aporta una mejora de la calidad educativa, del proceso de enseñanza-aprendizaje y por ende de los resultados de aprendizaje (Chacón y Pérez, 2011; Pinto et al., 2017; Talero y Elizabeth, 2012).

El uso de las diferentes herramientas tecnológicas posibilita, como señala Llisterri (2007), nuevas perspectivas y la integración de modo sistemático de la pronunciación de una L2 en contextos de autoaprendizaje, entornos de enseñanza no presenciales o semipresenciales. Es necesario no descuidar la enseñanza de la pronunciación al aprender una L2 y ser un componente más del diseño curricular que tiene la misma importancia que otros aspectos como la enseñanza de la gramática, el vocabulario, la cultura o las destrezas orales y escritas.

A pesar del aumento del interés por la enseñanza-aprendizaje de la pronunciación, es importante realizar más estudios en torno a este aspecto, ya que la mayoría de las investigaciones o estudios se realizan sobre otros aspectos de la lengua como pueden ser las destrezas escritas, la gramática, el vocabulario o la cultura. Además como Salinas (2005) expresa para que las instituciones de educación superior respondan al desafío de adaptarse a nuevas modalidades de enseñanza, deben promoverse experiencias innovadoras en las que la tecnología tenga un papel relevante.

Presentación del artículo: 4 de marzo de 2016

Fecha de aprobación: 26 de abril de 2016

Fecha de publicación: 30 de enero de 2017

Pinto, A.M., Sánchez, M.C., García-Peñalvo, F.J., y Cabezas, M. (2017). La modalidad semipresencial y la pronunciación de la lengua inglesa: Resultados de un modelo apoyado con TIC. RED. Revista de Educación a Distancia, 52. Consultado el (dd/mm/aaaa) en http://www.um.es/ead/red/52

La modalidad semipresencial y la pronunciación de la lengua inglesa: Resultados de un modelo apoyado con TIC. Ana $\mathrm{M}^{\mathrm{a}}$ Pinto, $\mathrm{M}^{\mathrm{a}}$ Cruz Sánchez, Francisco J. García-Peñalvo y Marcos Cabezas. 


\section{Referencias}

Allan, B. (2007). Blended Learning tools for teaching and training. London: Facet Publishing.

Arnal, J., Rincón, D. y Latorre, A. (1994). Investigación educativa. Fundamentos y metodología. Barcelona: Labor.

Bartolí, M. (2005). La pronunciación en la clase de lenguas extranjeras. Phonica, 1, 1-27

Buendía, L., Colás, P. y Hernández, F. (1997). Métodos de investigación en psicopedagogía. Madrid: McGraw-Hill.

Canale, M. (1983). De la competencia comunicativa a la pedagogía comunicativa del lenguaje. En M. Llobera (Coord.), Competencia comunicativa. Documentos básicos en la enseñanza de lenguas extranjeras (pp. 63-83). Madrid: Edelsa.

Canale, M. y Swain, M. (1980). Theoretical Bases of Communicative Approaches to Second Language Teaching and Testing. Applied Linguistics, 1, 1-47.

Cea D'ancona, M. A. (1998). Metodología cuantitativa: estrategias y técnicas de investigación social. Madrid: Síntesis.

Chacón, C. y Pérez, C. (2011). El podcast como innovación en la enseñanza del inglés como lengua extranjera. Pixel-Bit. Revista de Medios y Educación, 39, 41-54.

Coaten, N. (2003). Blended e-learning. Educaweb, 69. Disponible en: http://www.educaweb.com/esp/servicios/monografico/formacionvirtual/1181076.as p.

Colás, P. y Buendía, L. (1998). Investigación Educativa. Sevilla: Alfar.

Cole, J. y Foster, H. (2008). Using Moodle. Teaching with the Popular Open Source Course Management System. Sebastopol, California: O'Reilly Media.

Consejo Europeo (2001). Marco Común Europeo de Referencia de las Lenguas: Aprendizaje, enseñanza, evaluación. Madrid: MECD Grupo Anaya.

Cortés, M. (2002): Didáctica de la prosodia del español: la acentuación y la entonación. Barcelona: Edinumen.

Derwing, T.M. y Munro, M. (2005). Second Language Accent and Pronunciation Teaching: A Research-Based Approach. TESOL Quarterly, 39(3), 379-397.

Duffy, T. M., and Cunningham, D. J. (1996). Constructivism: Implications for the Design and Delivery of Instruction. En D. H. Jonassen (Ed.), Handbook of Research for Educational Communications and Technology (pp.170-198). New York: Macmillan Library Reference.

La modalidad semipresencial y la pronunciación de la lengua inglesa: Resultados de un modelo apoyado con TIC. Ana $\mathrm{M}^{\mathrm{a}}$ Pinto, $\mathrm{M}^{\mathrm{a}}$ Cruz Sánchez, Francisco J. García-Peñalvo y Marcos Cabezas. 
Dziuban, C. D., Hartman, J. L. y Moskal, P. D. (2004). Blended Learning. Educause Center for Applied Research, 7, 1-12.

García-Peñalvo, F. J. (2015). Cómo entender el concepto de presencialidad en los procesos educativos en el siglo XXI. Education in the Knowledge Society (EKS), 16(2), 6-12. doi:http://dx.doi.org/10.14201/eks2015162612

García-Peñalvo, F. J., \& Seoane-Pardo, A. M. (2015). Una revisión actualizada del concepto de eLearning. Décimo Aniversario. Education in the Knowledge Society, 16(1), 119-144. doi:10.14201/eks2015161119144

Graham, C. R. (2006). Blended Learning Systems. Definition, Current Trends, and Future Directions. En C. R. Graham y C. J. Bonk (Eds.), The Handbook of Blended Learning. Global Perspectives, Local Design (pp.3-21). San Francisco: Pfeiffer.

Gros, B., \& García-Peñalvo, F. J. (2017). Future trends in the design strategies and technological affordances of e-learning. In M. Spector, B. B. Lockee, \& M. D. Childress (Eds.), Learning, Design, and Technology. An International Compendium of Theory, Research, Practice, and Policy. Switzerland: Springer International Publishing.

Hernández, R., Fernández, C. y Baptista, P. (2010). Metodología de la investigación. México: McGraw Hill.

Honebein, P. C. (1996). Seven Goals for the Design of Constuctivist Learning Environment. En B. G. Wilson (Ed.), Constructivist learning environments: Case studies in instructional design (pp. 11-24). Englewood Cliffs, NJ: Educational Technology Publications.

Hymes, D. (1972). On Communicative Competence. En J. Pride y J. Holmes (Eds.), Sociolinguistics (pp. 269-293). Harmondsworth, UK: Penguin.

Iruela, A. (2004): Adquisición y enseñanza de la pronunciación en lenguas extranjeras. (Tesis doctoral). Barcelona: Universitat de Barcelona.

Iruela, A. (2007). Principios didácticos para enseñanza de la pronunciación en lenguas extranjeras. MarcoELE Revista de Didáctica, 4. Disponible en http://marcoele.com/principios-didacticos-para-la-ensenanza-de-la-pronunciacionen-lenguas-extranjeras

Kerlinger, F. N. (1985). Investigación del comportamiento. Técnicas y metodología. México: Nueva Editorial Interamericana.

Knuth, R. A. y Cunnigham, D. J. (1993). Tools for constrcutivism. En T. Duffy, J. Lowyck y D. Jonassen (Eds.), Designing environments for constructivist learning (pp.163-187). Berlín: Springer-Verlag.

Lado, R. (1964). Language Teaching: A Scientific Approach. New York: McGraw-Hill.

La modalidad semipresencial y la pronunciación de la lengua inglesa: Resultados de un modelo apoyado con TIC. Ana $\mathrm{M}^{\mathrm{a}}$ Pinto, $\mathrm{M}^{\mathrm{a}}$ Cruz Sánchez, Francisco J. García-Peñalvo y Marcos Cabezas. 
Larsen-Freeman, D. y Long, M. (1994). Introducción al estudio de la adquisición de segundas lenguas. Madrid: Gredos.

Larsen-Freeman, D. (2003). Techniques and Principles in Language Teaching. Oxford: Oxford University Press.

Levis, J.M. (2005). Changing Contexts and Shifting Paradigms in Pronunciation Teaching”. TESOL Quarterly, 39(3), 369-377.

Llisterri, J. (2007). La enseñanza de la pronunciación asistida por ordenador. En R. Mairal et al. (eds.), XXIV congreso internacional de AESLA. Aprendizaje de lenguas, uso del lenguaje y modelación cognitiva: Perspectivas aplicadas entre disciplinas (91-120). España, Madrid: UNED.

López, C. y Matesanz, M. (2009). Las plataformas de aprendizaje. Del mito a la realidad. Madrid: Editorial Biblioteca Nueva.

Man-Man, P. (2006). Developing Students' listening and speaking Skills Through ELT Podcasts. Educational Journal, 34(2), 115-134.

Osguthorpe, R.T. y Graham, C.R. (2003). Blended Learning Environments: Definitions and Directions. Quarterly Review of Distance Education, 4(3), 227-233.

Pastor, S. (2005). La enseñanza de segundas lenguas. En A. López y B. Gallardo (Ed.), Conocimiento y lenguaje (pp. 361-399). Valencia: PUV.

Pinto, A. M., Sánchez, M. C., y Palacios, B. (2014). Modelo Blended Learning para la enseñanza-aprendizaje del inglés en educación superior. En A. V. Martín (coord.) Blended Learning en educación superior. Perspectivas de innovación y cambio (121-142). Madrid: Editorial Síntesis.

Pinto, A.M., Sánchez, M.C. y García-Peñalvo, F.J. (2015). To be or not to be successful? That does not only depend on technology, but also on human factors. Journal of Cases on Information Technology, 17(1), 51-69.

Pinto, A.M., Sánchez, M.C. y García-Peñalvo, F.J. (2015). Developing a VLE to enable the innovative learning of English pronunciation. TEEM'15 3rd International Conference on Technological Ecosystems for Enhancing Multiculturality. Polytechnic of Porto-School of Engineering, Oporto, Portugal.

Pinto, A.M., Sánchez, M.C., y García-Peñalvo, F.J. (2016). Assessing the Effectiveness of Interactive and Collaborative Resources to Improve Reading and Writing in English. International Journal of Human Capital and Information Technology Professionals, 7(1), 66-85.

Pinto, A.M., Sánchez-Gómez, M.C., García-Peñalvo, F.J., y Casillas-Martín, S. (2017). Students' Perceptions and Attitudes Towards Asynchronous Technological Tools in Blended-Learning Training to Improve Grammatical Competence in English as a

La modalidad semipresencial y la pronunciación de la lengua inglesa: Resultados de un modelo apoyado con TIC. Ana $\mathrm{M}^{\mathrm{a}}$ Pinto, $\mathrm{M}^{\mathrm{a}}$ Cruz Sánchez, Francisco J. García-Peñalvo y Marcos Cabezas. 
Second Language. Computers in Human Behavior. In Press. doi:http://dx.doi.org/10.1016/j.chb.2016.05.071

Rice, W. (2011). Moodle 2.0 E-Learning Course Development. Birmingham: Packt publishing.

Richards, J. C. y Rodgers, T. S. (2001). Approaches and Methods in Language Teaching ( $2^{a}$ Ed.). Cambridge: Cambridge University Press.

Rosenberg, M. J. (2001). E-Learning Strategies for Delivering Knowledge in the Digital Age. New York: McGraw-Hill.

Ruiz, C. (2008). El Blended-learning: evaluación de una experiencia de aprendizaje en el nivel de postgrado. Investigación y Posgrado, 23(1), 11-36.

Salinas, J. (2005). La gestión de los entornos virtuales de formación. En Seminario Internacional: La Calidad de la Formación en Red en el Espacio Europeo de Educación Superior. España, Tarragona.

Sánchez, A. (1997). Los métodos en la enseñanza de idiomas: evolución histórica y análisis didáctico. Madrid: SGEL.

Santos, I. (1999). Lingüística Aplicada a la enseñanza-aprendizaje del español como lengua extranjera. Madrid: Arco Libros.

Sharpe, R., Benfield, G., Roberts, G. y Francis, R. (2006). The Undergraduate Experience of Blended Learning: A Review of UK Literature and Practice. Disponible http://www.heacademy.ac.uk/assets/documents/teachingandresearch/Sharpe_Benfie ld_Roberts_Francis.pdf

Talero, A., y Elizabeth, S. (2012). El podcast en la enseñanza de la lectoescritura. Revista Virtual Universidad Católica del Norte, 37, 9-26.

Tallei, J. I. y Coura, J. (2011). El uso del podcasts en la enseñanza secundaria. Revista de humanidades, tecnología e cultura, 1(1), 234-242.

Thorndike, R. M. (1997). Measurement and Evaluation in Psychology and Education. New York: MacMillan.

Thorne, K. (2003). Blended Learning: How to Integrate Online and Traditional Learning. London: Kogan

Usó, L. (2008). La enseñanza de la pronunciación en LE: algunas consideraciones. Phonica, 4, 104-130

Van Dalen, D. B. y Meyer, W. J. (1981). Manual de técnicas de la investigación educacional. Barcelona: Paidós.

La modalidad semipresencial y la pronunciación de la lengua inglesa: Resultados de un modelo apoyado con TIC. Ana $\mathrm{M}^{\mathrm{a}}$ Pinto, $\mathrm{M}^{\mathrm{a}}$ Cruz Sánchez, Francisco J. García-Peñalvo y Marcos Cabezas. Página 28 de 29 
La modalidad semipresencial y la pronunciación de la lengua inglesa: Resultados de un modelo apoyado con TIC. Ana $\mathrm{M}^{\mathrm{a}}$ Pinto, $\mathrm{M}^{\mathrm{a}}$ Cruz Sánchez, Francisco J. García-Peñalvo y Marcos Cabezas.

Página 29 de 29 